\title{
Computational haemodynamics of left coronary artery
}

\author{
J. V. Soulis ${ }^{1}$, T. M. Farmakis ${ }^{2}$, G. D. Giannoglou ${ }^{2}$, O. Faltsi ${ }^{3}$, D. \\ Sofialidis $^{3}$, J. Josipovic ${ }^{3}$ \& G. E. Louridas ${ }^{2}$ \\ 'Fluid Mechanics Division, Democrition University of Thrace, Xanthi, \\ Greece. $^{2}$

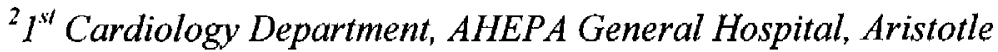 \\ University of Thessaloniki, Thessaloniki, Greece. \\ ${ }^{3}$ Simtec, Software \& Services Ltd, Thessaloniki, Greece.
}

\begin{abstract}
The purpose of our study was to quantitatively analyze the three-dimensional haemodynamics of the normal human Left Coronary Artery (LCA) tree. Physical parameters analyzed, amongst others, include: wall shear stress, wall stress and blood viscosity. The LCA tree includes: Left Anterior Descending, Left Circumflex Artery and their major branches. A 3D computer model of the normal LCA tree was adopted for subsequent numerical analysis. Discharges from distal branches were set proportional to the third power of the corresponding branch diameter. The blood was treated as a non-Newtonian fluid obeying to the power law. The governing mass, momentum and energy flow equations were solved using a previously validated numerical code. Low Wall Stress Gradients (WSG) and low Wall Shear Stress Gradients (WSSG) occur nearby to and at bifurcations, particularly at regions opposite the flow dividers, which are anatomic sites predisposed for atherosclerotic development. The Wall Stress (WS) decreases at distal LCA parts while the Wall Shear Stress (WSS) distribution reveals low values opposite to the flow dividers while increased values appear at distal tree parts. The viscosity distribution is highly affected from the blood flow conditions.
\end{abstract}




\section{Introduction}

The use of Computational Fluid Dynamics (CFD) to solve the governing Navier-Stokes equations of haemodynamics [1] is now reasonably practical using modern high-speed desktop computers. The solution of these equations can be readily validated by comparison with experimental data available in the general literature. Henceforth, computational analyzes supply information beyond that available from experimental studies.

Wall Shear Stress (WSS) [2]-[6] in vitro have shown their importance in atherogenesis, probably as local modulator of endothelial gene expression. The Wall Shear Stress Gradients WSSG in space as well as in time [7] have been suspected as being responsible for the atherogenesis. Published work referring to the importance of WSSG, contain experiments in vitro cellular cultures combined or not with CFD analysis. Despite all these efforts, the exact spatial role of WSSG in the Left Coronary Artery (LCA) tree still remains unclear. The importance of spatial wall stress [1] or wall static pressure is a relatively new concept which, for the last few years, is under investigation.

The aim of the present study was to numerically analyze the 3D flow in the normal human LCA tree, applying a finite-volume technique in order to investigate the spatial Wall Stress (WS), Wall Stress Gradients (WSG), WSS, WSSG and viscosity distribution in these arteries. In order to expand the investigation, a realistic model of typical vessel geometry of the left coronary artery system including the left main, left anterior descending, and circumflex arteries is presented. The results shed light into the $3 \mathrm{D}$ nature of the flow patterns and quantify the distribution of WSG and WSSG particularly in regions close and opposite the flow divider in proximal and distal areas of the LCA tree.

\section{Materials and methods}

\subsection{Geometry}

Dodge JT Jr et al [8],[9] published experimental measurements of the intrathoracic spatial location of specified coronary segments on the normal human heart. In these studies, which were based on 83 coronary angiographies taken from normal subjects, the exact intrathoracic location and course of each one of the 23 arterial segments and branches, which are commonly used by the physician to describe the localization of coronary disease, were recorded. For current research work purposes, a 3D computational model of the (LCA) tree was constructed. This LCA tree model includes, Figure 1, the Left Main Coronary Artery (LMCA), the Left Anterior Descending Artery (LAD), the Left Circumflex Artery (LCxA) and their main branches.

\subsection{Mesh generation}

All numerical technique analyses require the discretization of the flow field into finite volumes or cells [10]. The anatomic configuration of the LCA tree is 
highly complex. Therefore, the application of a non-structured computational grid is a necessity. At all, 40843 grid nodes were utilized, see Fig. 1, giving rise to 164041 computational tetrahedra requiring $133 \mathrm{MB}$ of physical memory.

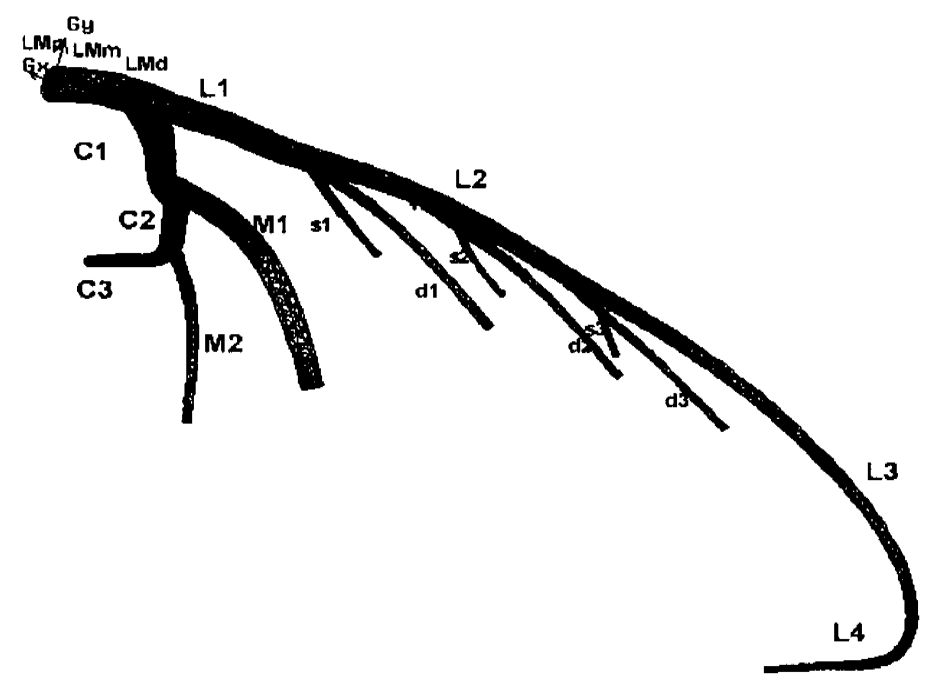

Figure 1: Geometry of the unstructered mesh of the tested human LCA tree.

Figure 2 show details of the non-structured grid of the LCA tree. The number of tetrahedra used in the current analysis was considered to be satisfactory for the heamodynamic analysis. It should be noticed that the adequate calculation of WSG and WSSG variables in complex geometry configurations, such as the LCA tree, requires careful adaptation of the computational grid, compared to the calculation based on grid adapted from velocity components. The criterion of grid adaptation based on WSSG was used for grid node densing.

\subsection{Equations and boundary conditions}

All mesh and flow data were transferred into the main CFD solver FLUENT (Fluent Inc., Lebanon, NH). The numerical code solves the governing NavierStokes flow equations. In their generality, these equations solve the mass, momenta and energy conservation. The numerical procedure uses finite volume method to achieve solution. The assumptions made about the flow are that it is: three-dimensional, unsteady and laminar, while the arterial vessel walls are inelastic and impermeable. The blood is treated as non-Newtonian fluid obeying to the non-Newtonian power law [1]. According to this law the fluid shear stress $\tau\left(\mathrm{N} / \mathrm{m}^{2}\right)$ is calculated, 


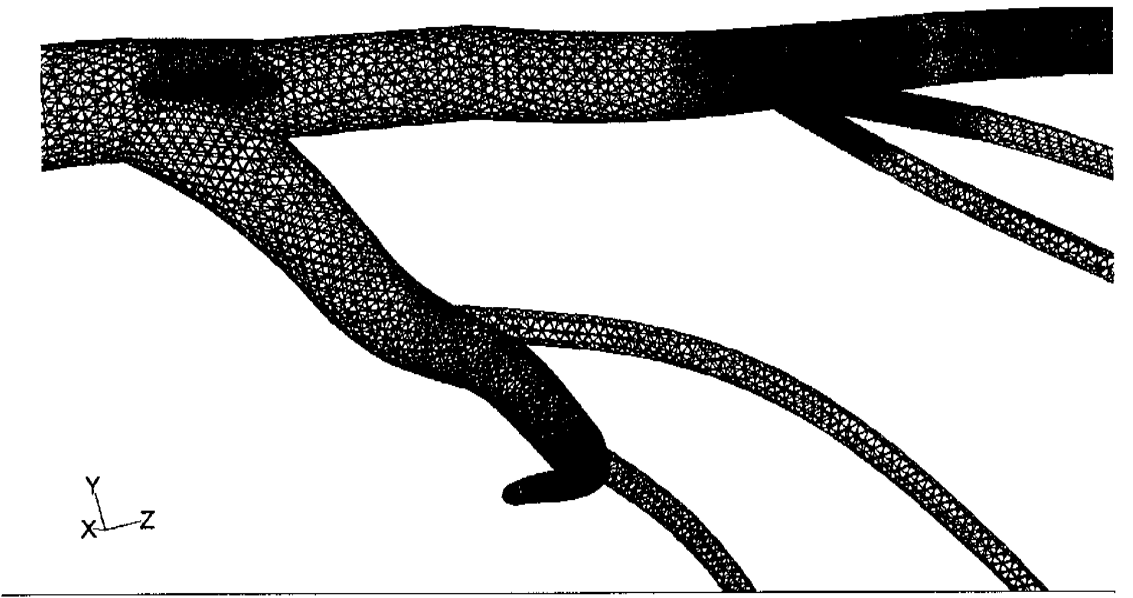

Figure 2: Non-structured computational grid of the LCA used for analysis

$$
\tau=[\eta(\dot{S})] \dot{S}
$$

with

$$
\begin{aligned}
& \eta(\dot{S})=k e^{\frac{T_{0}}{T}} \dot{S}^{n-1} \\
& \dot{S}=\frac{\partial u_{i}}{\partial x_{j}}+\frac{\partial u_{j}}{\partial x_{i}}
\end{aligned}
$$

where $k, n$ are parameters [1], while $T$ and $T_{a}$ refer to local and reference temperature, respectively. The flow governing equations are,

$$
\frac{\partial \rho}{\partial t}+\frac{\partial}{\partial x_{i}}\left(\rho u_{i}\right)=S_{m}
$$

$t$ (s) is the time, $u_{i}(\mathrm{~m} / \mathrm{s})$ are the velocity components, along the $x_{i}(\mathrm{~m})$ axes, while $S_{m}$ is the added or subtracted mass of fluid in the flow field. The repetition of index $i$ denotes summation. The conservation of momentum along the $i$ direction is written,

$$
\frac{\partial}{\partial t}\left(\rho u_{i}\right)+\frac{\partial}{\partial x_{j}}\left(\rho u_{i} u_{j}\right)=-\frac{\partial p}{\partial x_{i}}+\frac{\partial \tau_{i j}}{\partial x_{j}}+\rho g_{i}+F_{i}
$$


similarly for the other directions. Here, $p\left(\mathrm{~N} / \mathrm{m}^{2}\right)$ is the static pressure, $\tau_{i j}\left(\mathrm{~N} / \mathrm{m}^{2}\right)$ is the shear stress tensor, $\rho g_{i}\left(\mathrm{~N} / \mathrm{m}^{3}\right)$ and $F_{i}\left(\mathrm{~N} / \mathrm{m}^{3}\right)$ are gravity and externally acting forces, respectively. The blood velocity is assumed to be uniform at the orifice of LMCA, which corresponds to the flow inlet. Rest flow conditions were applied at the inlet corresponding to $0.17 \mathrm{~m} / \mathrm{s}$. Flow discharges are set analogous to the third power of the branching vessel radius. These discharges are computed at the exit of branching vessels. On the endothelium, the non-slip boundary condition requires that all flow velocity components are equal to zero. After an initial convergence of the flow, a grid adaptation is performed. For a typical satisfactory convergence solution a total of 2000 time steps were required. Convergence is achieved when all velocity component changes from iteration to iteration were less than $10^{-6}$.

\section{Results}

\subsection{Wall stress and wall stress gradient}

Figure 3 shows the WS distribution in the LCA tree. The WS varies in space and low values of them are frequently co-exist with high values. Low WS values occur at the proximal bifurcation. Figure 4 shows the contours of WSG for the LCA tree. High WSG values coexist with low WSG values, as it is evident from above figure. Computational results indicate that on the LCA tree and for the regions shown in Fig. 4 the WSG range from 68 to $38400 \mathrm{~N} / \mathrm{m}^{3}$. At proximal regions of the LCA, the WSG values are always lower to those occurring at the distal parts of the tree. This is due to increased velocity resulted from increased vessel tapering. The dark color indicates regions of low WSG values.

\subsection{Wall shear stress and wall shear stress gradient}

Figure 5 shows the contours of WSS in N/m² for the LCA tree. Low values, in the range $0.68-5.09 \mathrm{~N} / \mathrm{m}^{2}$, appear in regions opposite to the flow divider. At distal tree parts the WSS values increase substantially. Figure 6 shows the contours of WSSG, in N/m $\mathrm{m}^{3}$, at the proximal bifurcation region of the LCA tree. High WSSG values occur at the flow divider. In this region these values extend to more than $1200.0 \mathrm{~N} / \mathrm{m}^{3}$. Low WSSG values occur opposite to the flow divider. Their range is 7.3 to $348.07 \mathrm{~N} / \mathrm{m}^{3}$. Low values of this physical derivative are responsible for atherosclerosis, [1], [6].

\subsection{Viscosity}

Finally, Fig. 7 shows the contours of molecular viscosity in $\mathrm{kg} / \mathrm{m}$-s for the LAD. Dark color indicates regions of low viscosity values. High viscosity values appear in regions opposite to the main flow divider. 
194 Simulations in Biomedicine $V$

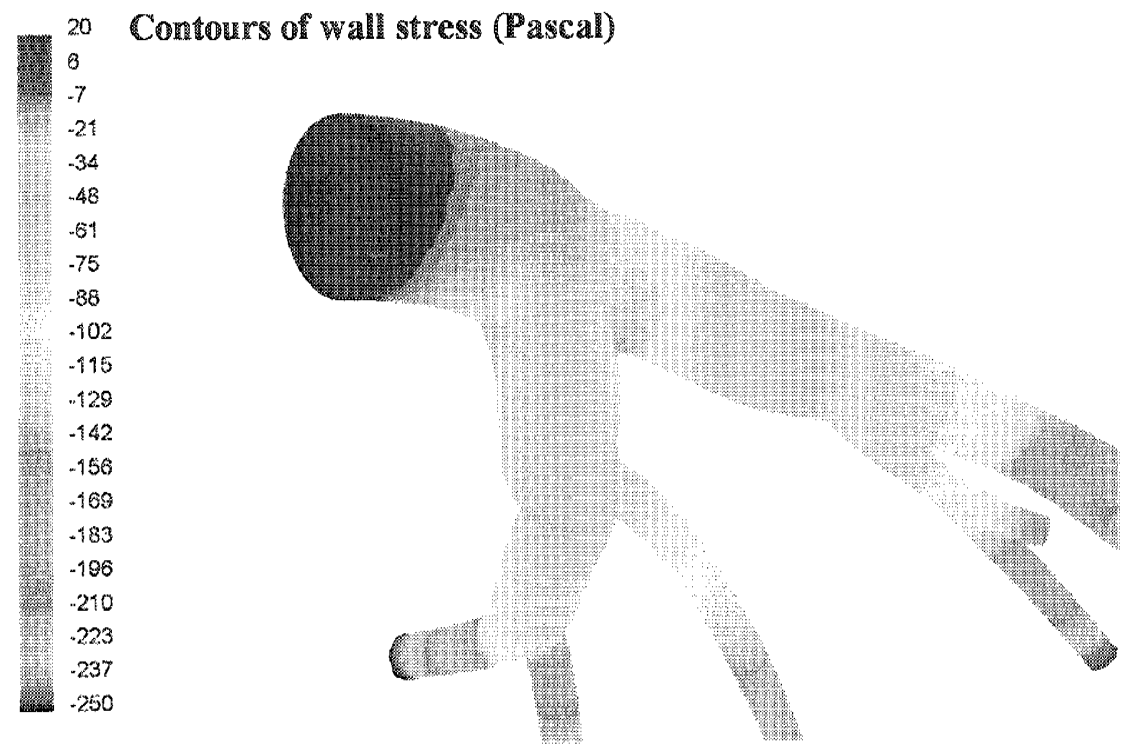

Figure 3: Contours of WS for the LAD. WS values decrease at distal regions.

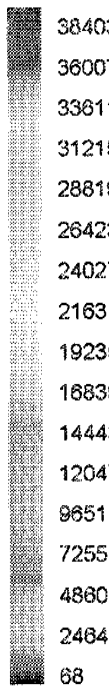

\section{Contours of wall stress gradient (Pascal/m)}

36007

33011

31245

28819

26423

24027

21631

19235

16839

14443

12047

9651

7255

4860

2464

58

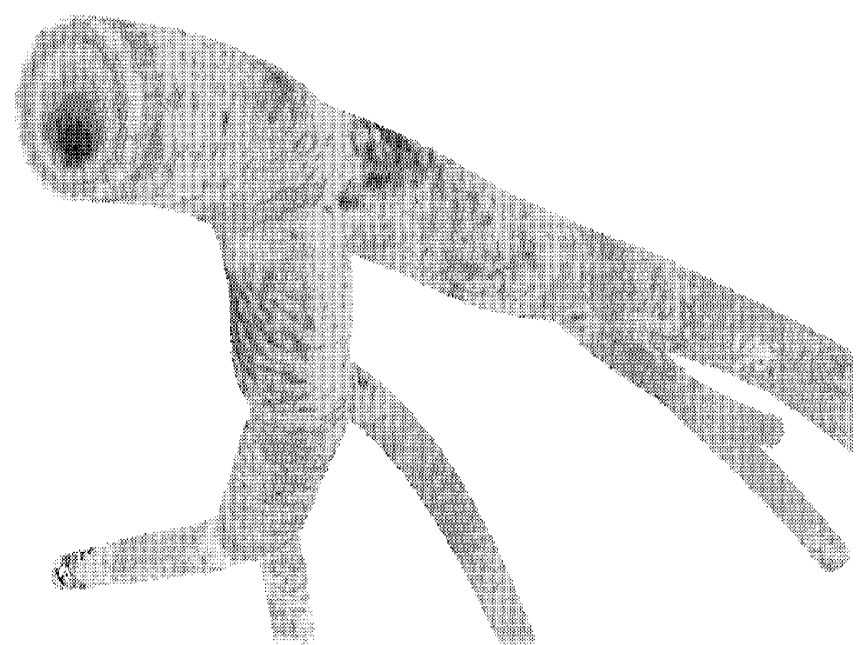

Figure 4: Contours of WSG for the LCA tree. Dark color indicates regions of low WSG values. 

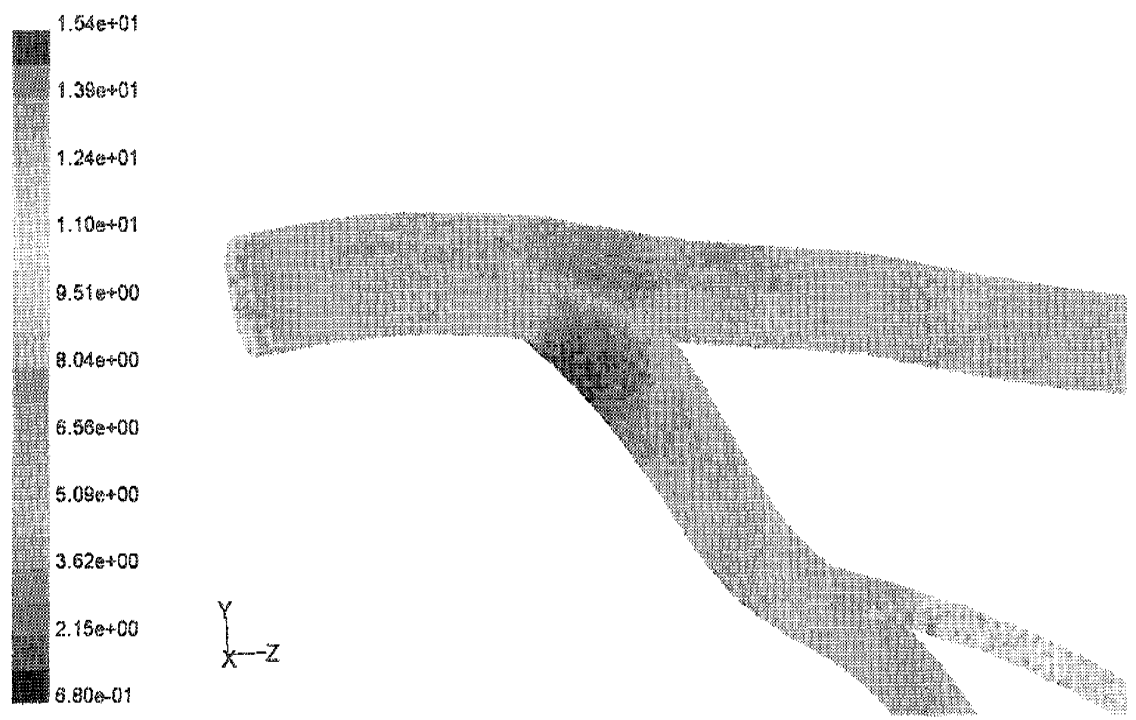

Figure 5: Contours of WSS $\left(\mathrm{N} / \mathrm{m}^{2}\right)$ for the LCA tree.

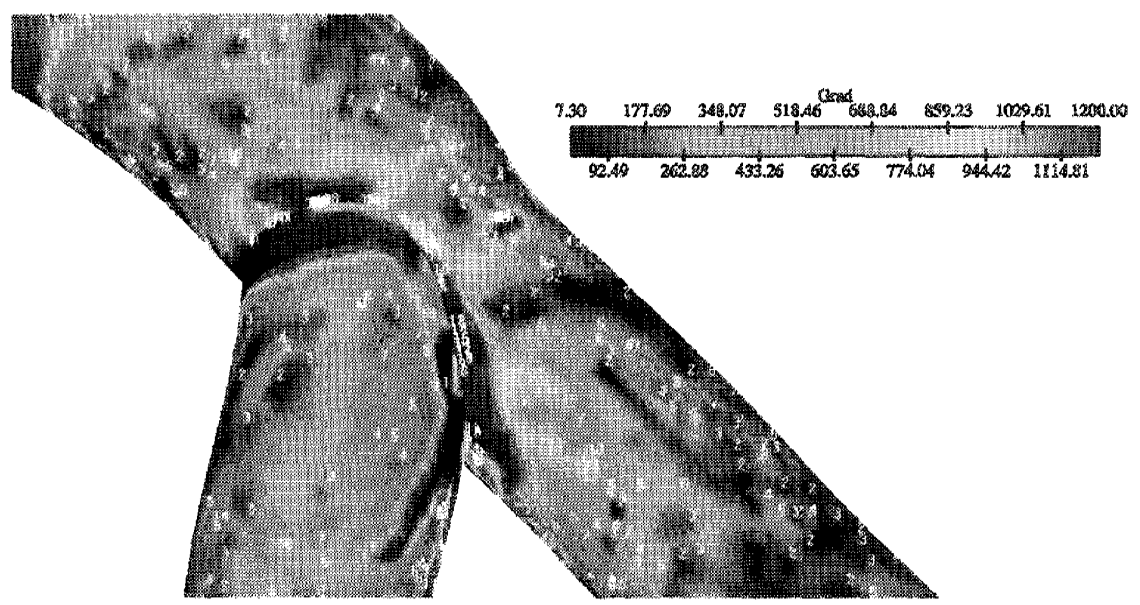

Figure 6: Contours of WSSG $\left(\mathrm{N} / \mathrm{m}^{3}\right)$ for the LCA tree (LMCA main bifurcation). 


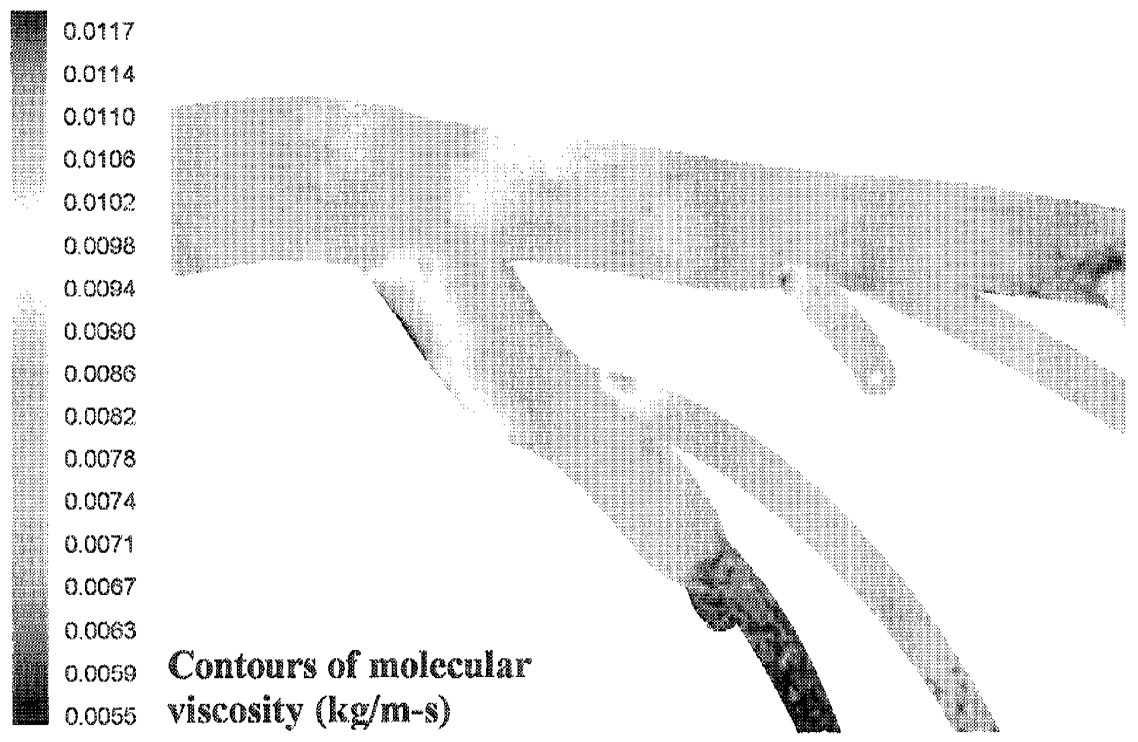

Figure 7: Contours of molecular viscosity for the LCA.

The viscosity distribution is highly affected from the blood flow conditions. High viscosity values appear in regions opposite to the main flow divider. At distal parts, where the shear rate is high, the viscosity values are low.

\section{Discussion}

Via the application of the CFD, as it is applied to human coronary artery data, is possible to quantitatively analyze the local hemodynamic parameters, particularly the WSG as well as WSSG, in order to elucidate the blood flow patterns in regions where atherosclerosis usually appears. Lesion-prone areas are bifurcations and trifurcations.

Despite the fact that the flow basically remains laminar, in regions of abrupt geometry change the flow pattern is characterized by: a) flow separation, b) flow reversal and c) reattachment of blood flow lines with the arterial wall. The appearance of haemodynamic disturbances has been correlated with the orientation and displacement of endothelial cells [1],[12],[13], as well as the metabolic function changes. It has been proved that, when the formed elements of blood are trapped within the recirculation zone, they are allowed to adhere to endothelial cells in regions of low wall shear stress.

As it is evident from the WSG as well as the WSSG distribution of the LMCA bifurcation, Figs. 4 and 6, the low WSG and WSSG regions are located at the sidewalls opposite the flow divider. This distribution is in accordance to the localization of atherosclerotic lesions in this area [1],[11],[14]. Thus, there is strong evidence that these values are possibly correlated to the localization of atherosclerotic lesions. High WSG amd WSSG values are encountered at the 
flow divider. All contours presented in the current research work show their magnitude and not their spatial direction. In region near the vessel flow divider, there is a large variation from low to high gradient values. Moreover, from the overall examination of the above contours, it is also evident that WSG present high values in the distal regions of the LCA, where the magnitude of the mean flow velocity is relatively higher, due to the vessel tapering. On the contrary, lower WSG and WSSG values appear in the proximal LCA regions. This low WSG values distribution is in accordance to the frequent localization of atherosclerotic lesion in the proximal LCA regions. This localization is well known to clinical cardiologist. It is also well documented from numerous pathological studies. This fact further supports the possible correlation between low WSG and the localization of atherosclerosis. High curvature geometries of vessels or bifurcation or trifurcation regions are suspected to be prone to atherosclerosis. These "vulnerable" regions, presenting flow disturbances with recirculation and flow separation, usually develop low WSG and WSSG values.

\section{Conclusion}

The current study presents the 3D haemodynamics solution of human left coronary artery non-Newtonian blood flow. The analysis in this arterial segment refers to the spatial WS, WSS, WSG, WSSG and viscosity distribution and elucidates the blood flow patterns in regions where atherosclerosis usually appears. Low WSG and WSSG occur nearby to and at bifurcations, particularly at regions opposite the flow divider, which are anatomic sites predisposed for atherosclerotic development. In general, low WSG and WSSG appear in the proximal regions of the LCA tree, where atherosclerosis frequently occurs. At distal segments the WSG as well as the WSSG increase substantially, due, perhaps, to increased velocity resulted from increased vessel tapering. CFD analysis is a useful tool to determine the haemodynamic distribution of complex wall stress patterns, elucidating the process of genesis and progression of atherosclerosis in human.

\section{References}

[1] Giannoglou G.D., Soulis J.V., Farmakis T.M., Farmakis D.M., Louridas G.E., Haemodynamic factors and the important role of local low static pressure in coronary wall thickening, Int $J$ Cardiol., 86(1), 27, in press, 2002.

[2] Asakura T., Karino T., Flow patterns and spatial distribution of atherosclerotic lesions in human coronary arteries. Circ Res, 66(4), pp.1045-66, 1990.

[3] Gibson C.M., Diaz L., Kandarpa K., Sacks F.M., Pasternak R.C., Sandor T., Feldman C., Stone P.H., Relation of vessel wall shear stress to atherosclerosis progression in human coronary arteries, Arterioscler Thromb, 13(2), pp. 310-5, 1993. 
[4] Krams R., Wentzel J.J., Oomen J.A., Vinke R., Schuurbiers J.C., de Feyter P.J., Serruys P.W., Slager C.J., Evaluation of endothelial shear stress and $3 \mathrm{D}$ geometry as factors determining the development of atherosclerosis and remodeling in human coronary arteries in vivo. Combining 3D reconstruction from angiography and IVUS (ANGUS) with computational fluid dynamics, Arterioscler Thromb Vasc Biol, 17(10), pp. 2061-5, 1997 .

[5] Nagel T., Resnick N., Dewey C.F. Jr, Gimbrone M.A. Jr., Vascular endothelial cells respond to spatial gradients in fluid shear stress by enhanced activation of transcription factors, Arterioscler Thromb Vasc Biol., 19(8), pp. 1825-34, 1999.

[6] Tardy Y., Resnick N., Nagel T., Gimbrone M.A. Jr, Dewey C.F. Jr., Shear stress gradients remodel endothelial monolayers in vitro via a cell proliferation-migration-loss cycle, Arterioscler Thromb Vasc Biol., 17(11), pp. 3102-6, 1997.

[7] White C.R., Haidekker M., Bao X., Frangos J.A., Temporal gradients in shear, but not spatial gradients, stimulate endothelial cell proliferation. Circulation, 103(20), 2508-13, 2001.

[8] Dodge J.T. Jr, Brown B.G., Bolson E.L., Dodge H.T., Lumen diameter of normal human coronary arteries. Influence of age, sex, anatomic variation, and left ventricular hypertrophy or dilation, Circulation, 86(1), pp. 232-46, 1992.

[9] Dodge J.T. Jr, Brown B.G., Bolson E.L., Dodge H.T., Intrathoracic spatial location of specified coronary segments on the normal human heart. Applications in quantitative arteriography, assessment of regional risk and contraction, and anatomic display, Circulation, 78(5 Pt 1), pp.1167-80, 1988.

[10] Kleinstreuer C., Engineering Fluid Dynamics. An Interdisciplinary Systems Approach: Cambridge University Press, 1997.

[11] Friedman M.H., Deters O.J., Mark F.F., Bargeron C.B., Hutchins G.M., Arterial geometry affects hemodynamics. A potential risk factor for atherosclerosis. Atherosclerosis, 46(2), pp. 225-31, 1983.

[12] Yoshizumi M., Kurihara H., Sugiyama T., Takaku F., Yanagisawa M., Masaki T., Yazaki Y., Hemodynamic shear stress stimulates endothelin production by cultured endothelial cells, Biochem Biophys Res Commun 1989, 161(2), pp. 859-64, 1989.

[13] Honda H.M., Hsiai T., Wortham C.M., Chen M., Lin H., Navab M., Demer L.L., A complex flow pattern of low shear stress and flow reversal promotes monocyte binding to endothelial cells. Atherosclerosis, 158(2), pp. 385-90, 2001.

[14] Halon D.A., Sapoznikov D., Lewis B.S., Gotsman M.S., Localization of lesions in the coronary circulation, Am J Cardiol., 52(8): pp. 921-6, 1983. 